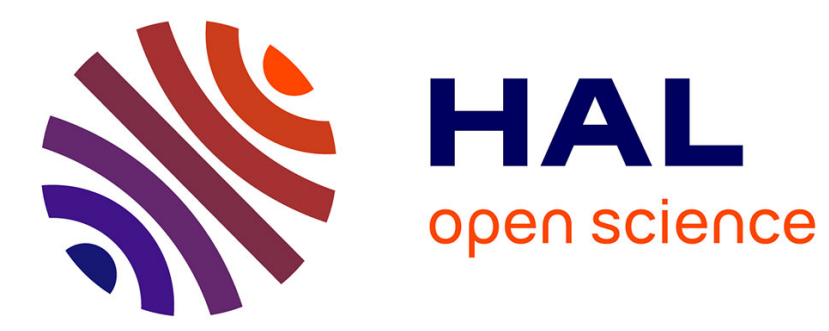

\title{
Perceptual Thresholds for Acoustical Guitar Models
}

\author{
J. Woodhouse, E. K. Y. Manuel, L. A. Smith, A. J. C. Wheble, Claudia Fritz
}

\section{To cite this version:}

J. Woodhouse, E. K. Y. Manuel, L. A. Smith, A. J. C. Wheble, Claudia Fritz. Perceptual Thresholds for Acoustical Guitar Models. Acta Acustica united with Acustica, 2012, 98 (3), pp.475-486. 10.3813/AAA.918531 . hal-01461755

\section{HAL Id: hal-01461755 https://hal.science/hal-01461755}

Submitted on 1 Sep 2021

HAL is a multi-disciplinary open access archive for the deposit and dissemination of scientific research documents, whether they are published or not. The documents may come from teaching and research institutions in France or abroad, or from public or private research centers.
L'archive ouverte pluridisciplinaire HAL, est destinée au dépôt et à la diffusion de documents scientifiques de niveau recherche, publiés ou non, émanant des établissements d'enseignement et de recherche français ou étrangers, des laboratoires publics ou privés. 


\title{
Perceptual Thresholds for Acoustical Guitar Models
}

\author{
J. Woodhouse ${ }^{1)}$, E. K. Y. Manuel ${ }^{1)}$, L. A. Smith ${ }^{1)}$, A. J. C. Wheble ${ }^{1)}$, C. Fritz ${ }^{2)}$ \\ 1) Cambridge University Engineering Department, Trumpington St, Cambridge CB2 1PZ, UK. jw12@ cam.ac.uk \\ 2) LAM, Institut Jean le Rond d'Alembert, UMR 7190, CNRS / UPMC Univ Paris 06, 11 rue de Lourmel, Paris, \\ France
}

\begin{abstract}
Summary
Synthesised acoustic guitar sounds based on a d etailed physical model are used to provide input for psycho-acoustical testing. Thresholds of perception are found for changes in the main parameters of the model. Using a three-alternative forced-choice procedure, just-noticeable differences are presented for changes in frequency a nd damping of the modes of the guitar body, a nd also for changes in the tension, bending stiffness and damping parameters of the strings. These are compared with measured data on the range of variation of these parameters in a s election of guitars.
\end{abstract}

\section{Introduction}

Assessments of the quality of musical instruments can be influenced by many factors, including appearance and ergonomic considerations, but the final judgement will presumably always relate to some extent to perceived quality of sound. Instrument makers want to know how to link particular changes in physical structure to their effect on perceived sound, so that they can control and improve the quality of their instruments. A useful first step in mapping out these links, which lends itself to relatively uncontroversial psychoacoustical testing, is to establish the smallest change of each structural parameter that produces an audible effect under the most favourable listening conditions. A comprehensive set of such just-noticeable differences (JNDs) would give information about the relative sensitivity of the sound to the different parameters under the control of an instrument maker, without the complications of trying to describe the nature of the changes in sound. Such descriptions are important, of course, but they require different methods to study them and can raise more difficult challenges in experimental methodology. This paper concentrates on the first stage, finding JNDs.

A recent research project [1] has investigated some JNDs for violin acoustics. While that work was going on, a series of undergraduate projects was used to perform a parallel investigation of the acoustical guitar, using similar psychoacoustical methodology. This paper summarises the main findings of those projects. As well as psychoacoustical results, measurements on a variety of guitars will be presented to provide information about the actual range of variation in the parameters investigated.
In one important respect the guitar is very different from the violin. In the violin, the player can produce a sustained sound by feeding energy into the vibrating string from the bow. This involves nonlinear interactions (from the friction force at the bow-string contact: see e.g. [2]) that make theoretical modelling difficult, and it also means that the player can influence the details of the sound of each note throughout its duration. The guitar is quite different: the instrument is, to a good approximation, a linear vibrating system. The player starts a particular note with control over the details of the pluck, but once the string has been released the subsequent sound is not under the player's control. (This study excludes any use of vibrato.)

This distinction leads to a difference in the way that sounds for psychoacoustical testing can most usefully be generated. For the violin, it is remarkably hard to synthesise sound that is convincingly realistic because of the allpervading influence of the human performer. Instead, a hybrid strategy was adopted for the psychoacoustical tests, in which real playing was combined with digital-filter emulation of the vibration response of the violin body. The fluctuating force exerted by a vibrating string on the violin bridge during normal playing was measured with an embedded piezoelectric sensor, and recorded. It was argued that, to a useful first approximation, a given bow gesture on a given string will produce essentially the same force waveform regardless of the acoustical response of the particular violin body. For example, during a steady note the player will ensure that the string vibrates in Helmholtz motion, producing a sawtooth force waveform at the bridge with relatively subtle modifications due to the bowing position and force, and the string parameters such as damping and bending stiffness [2].

It was thus argued that the main effects of a particular violin body on the perceived sound should arise from 
the filtering of this bridge-force signal by the linear vibration response and sound radiation characteristics of the violin. Linear vibration response can be analysed into a sum of modal contributions, and can then relatively easily be emulated by a suitable digital filter. So, for example, to investigate the JND for a shift in frequency of one particular vibration mode of the body, a series of sound files could be generated using the same recorded bridge force signal, modified by a range of digital filters initially calibrated against a particular violin body but with the one desired mode frequency systematically altered. The use of recorded bridge-force signal from a human performer gets around the problem of realism, without giving the player any opportunity to alter their bowing in response to the parameter being varied because the same input signal is used in every case.

For the guitar, this approach will not work convincingly. Part of the sound quality of a guitar note comes from the frequency-dependent decay rates of the different overtone components of the sound. These are determined by interaction of the string and the guitar body through the coupling at the bridge. The vibration response of a guitar body could be emulated by digital filter just as easily as a violin body, but if the hybrid approach were to be used for the guitar, the mechanical coupling of string to body would be missing and it is likely that a crucial part of the influence of the body acoustics on the sound would be lost. Luckily, the issue of non-realism of synthesised sound is much less challenging for the guitar. A synthesis model for guitar notes based on a detailed physical model has been described previously [3, 4], and this has been found to produce quite plausible sounds. Indeed, several participants in the studies to be described here expressed surprise when told that they were hearing synthesised sounds. In consequence, the JND studies for guitars were carried out entirely using synthesised sounds.

There is very little existing literature that is directly relevant to this study. While a lot has been written about the vibration and sound radiation characteristics of guitar bodies, published psychoacoustical studies based on the modelling are very rare: the only example seems to be the doctoral work of Wright [5]. There is, however, some literature relating to guitar strings, specifically to the perception of inharmonicity associated with bending stiffness. Two separate studies have been published by Järveläinen and co-workers $[6,7]$ using synthesised sounds of differing degrees of realism to obtain thresholds for discrimination between harmonic and inharmonic sounds. They found that thresholds for highly idealised sounds were lower than for more realistic sounds including the initial transient of a guitar pluck, presumably because of some degree of informational masking in the more complex sounds.

The outline of this paper is as follows. The synthesis model is briefly described, and the parameters to be explored in JND studies are defined. The technical details of the psychoacoustical test procedure are then given, followed by the acoustical test methods for establishing physical parameter values for the guitar body and for the strings investigated. Results are then presented, first for the properties of the guitar body. Several tests will be presented in which modal frequencies are shifted: all together, or confined to particular frequency bands. The influence of the choice of musical passage for the test stimulus is also discussed. After this, a more restricted study was undertaken in which the modal damping factors were scaled. The final section of test results concerns properties of the strings, especially the damping properties. These results will be related to the perception of "new" versus "old" strings by a change in their characteristic sound, in the light of measurements of what actually changes as steel-cored guitar strings age.

\section{Outline of synthesis model}

The synthesis model to be used here is primarily aimed at achieving an accurate representation of the coupled mechanical vibration of the strings and guitar body. It does not attempt a comparably full treatment of the details of the player's pluck gesture, or of the sound radiation behaviour: these are both taken into account in a simple way only, as will be described shortly. The input to the model is a set of parameter values describing separately the behaviour of the guitar body and the strings. For each body mode four things are required: the natural frequency, the modal damping factor, the modal amplitude at the position on the bridge where the string is coupled, and an angle describing the orientation of motion at that coupling point. The modal amplitude can be equivalently expressed via an effective mass: the amplitudes deduced by the techniques of experimental modal analysis correspond to mass-normalised modes (see for example Ewins [8]), and the square of the measured amplitude at a given point is the inverse of the effective mass for representing that mode at that point by a mass-spring-damper combination. The angle of motion at the coupling point is needed so that both polarisations of string motion can be included in the synthesis.

Ideally, the amplitudes and angles of each body mode would be separately determined at the six positions on the bridge saddle where the strings make contact. This would be possible but laborious, and for the present study it has not been done. The body modal parameters are deduced from a measurement of the input admittance (also called the driving point mobility) at a position between the 5th and 6th strings (the two tuned to the lowest frequencies). The same body response is used for all strings in the synthesis process. This position was chosen mainly because it is easier to perform the measurements necessary to establish the modal angles at the ends of the bridge rather than in the middle for practical reasons of access. Other positions on the bridge would of course give the same natural frequencies and modal damping factors, but with different amplitudes and angles. Since the aspects of body behaviour studied here involve only frequencies and damping, one might hope that the conclusions would not be significantly changed by a more thorough synthesis allowing different body behaviour for each string. 
The string is treated as a continuous system rather than being described in modal terms: this was shown to give a more efficient strategy for computing the coupled string/body motion with correct allowance for the very different damping levels of the two systems, which causes the damping of the coupled system to be strongly nonproportional [3]. A given string is specified by its length $L$, tension $T$, mass per unit length $m$, bending stiffness $E I$, and a measure of its damping. The bending stiffness is expressed as the product of the Young's modulus $E$ and the second moment of area $I$, which for a homogeneous monofilament string of diameter $d$ is given by

$$
I=\pi d^{4} / 64 \text {. }
$$

Inharmonicity due to bending stiffness is often expressed in terms of the dimensionless parameter $B$ that appears in the approximate expression for the frequency of the $n$th overtone,

$$
f_{n} \approx n f_{0} \sqrt{1+B n^{2}}
$$

where $f_{0}$ is the fundamental frequency. In terms of the notation introduced here,

$$
B=\frac{E I \pi^{2}}{T L^{2}}
$$

For overwound strings, as normally used for the lower strings of the guitar, the effective value of $E I$ or $B$ cannot be reliably predicted, and must be measured.

Musical strings in general have been shown to exhibit strongly frequency-dependent damping, and the measured behaviour can be fitted to a three-parameter model proposed by Valette [9]. The loss factor of the $n$th mode is assumed to take the form

$$
\eta_{n}=\frac{T\left(\eta_{F}+\eta_{A} / \omega_{n}\right)+E I \eta_{B}(n \pi / L)^{2}}{T+E I(n \pi / L)^{2}}
$$

The damping is lowest in the mid range of frequencies, with a minimum level set by $\eta_{F}$. At very low frequencies the damping increases, an effect attributed by Valette to air damping and quantified by the parameter $\eta_{A}$. At high frequencies the damping increases again, because energy dissipation associated with the complex bending stiffness of the string comes to dominate, especially for the polymerbased strings of classical guitars. This effect is characterised by the parameter $\eta_{B}$.

The chosen synthesis method works in the frequency domain, then uses an inverse FFT to obtain the time response. Full details are given in [3, 4]. The string and body are coupled at the bridge using the standard point-coupling formula

$$
Y_{\text {coup }}^{-1}=Y_{\text {body }}^{-1}+Y_{\text {str }}^{-1},
$$

where $Y_{\text {coup }}, Y_{\text {body }}, Y_{\text {str }}$ are the $2 \times 2$ matrices of admittance at the bridge notch for the coupled system, the body alone and the string alone, respectively. Each synthesised note begins with an idealised pluck, which is a step function of force as the finger or plectrum loses contact with the string. The string is assumed to be released from rest at the instant of the note starting. Advantage is taken of the reciprocity principle: it is convenient to apply the force step at the bridge and calculate the response at the point on the string where the pluck acts. As well as the plucking position on the string, it is possible to specify an angle of pluck (which determines the mix of the two polarisations of string motion excited). The player's finger or plectrum will have a finite size, and this will have the effect of a low-pass filter on the excitation of string modes. This is represented in a simple way by a frequency-domain Gaussian filter proportional to $\exp \left(-\omega^{2} / K^{2}\right)$. Here, $K=v / h$ where $v=\sqrt{T / m}$ is the wave speed of the string and the width $h$ was given the value $7.5 \mathrm{~mm}$ in order to obtain a reasonably realistic sound.

The synthesis model calculates the transient waveform of acceleration at the coupling point between the string and body. A full model would then need to calculate the body motion at all other positions, and perform a rather complicated numerical calculation to give the resulting sound radiation. The present model uses a severely simplified approach: the computed bridge acceleration is regarded as being applied to a spherical radiating body of radius $a$, vibrating in the symmetric breathing mode only. For that problem, the sound pressure in the far field is given simply by filtering the acceleration waveform with a low-pass filter that, apart from an overall scale factor, takes the form

$$
R(\omega)=\frac{\mathrm{i} k a}{1+\mathrm{i} k a}=\frac{\mathrm{i} \omega / \omega_{c}}{1+\mathrm{i} \omega / \omega_{c}},
$$

where $k=\omega / c$ is the wavenumber for sound in air, $c$ is the speed of sound, and $\omega_{c}=c / a$ is the filter roll-off frequency. Setting this roll-off at $250 \mathrm{~Hz}$, corresponding to a sphere radius around $0.2 \mathrm{~m}$, proved to give satisfactorysounding results.

This synthesis procedure has been implemented in Matlab, in a program that allows the user to specify a musical fragment entered in a form of guitar tablature. The resulting "tune" is saved as a sound file.

\section{Experimental methodology}

\subsection{Psychoacoustical tests}

Using synthesised sounds generated by this method, experiments were conducted to give some JND information relating to the major parameters. For the body model, experiments were done in which all the mode frequencies were shifted by a given factor, and also groups of modes in particular frequency bands were shifted, leaving the other modes unchanged. In a separate pair of tests, the damping factors of all modes were modified by a given factor, either upwards or downwards from the reference values in case there was an asymmetry of perception. For the string model, the bending stiffness and the damping parameters $\eta_{F}, \eta_{B}$ were changed. The parameter $\eta_{A}$ was found to have so little influence on the chosen test sound that it was not 
possible to obtain a meaningful JND for changes to its value. A general comment should be noted: all the tests to be reported here were constrained by the time limits imposed by the format of undergraduate projects. In some cases there is no doubt that more could have been learned had more time been available, but it is felt that the results have value even with these limitations.

JND thresholds were estimated using a three-alternative forced-choice procedure (3AFC). This is not the only possible procedure that could have been used, but it had already been chosen for the violin studies and software to implement $3 \mathrm{AFC}$ was conveniently to hand. A threedown one-up adaptive tracking rule was used that estimated the $79 \%$ correct point on the psychometric function [10]. It should be noted that this is the definition assumed for "JND" throughout this paper: it is a rather conservative value, significantly higher than that which would correspond to the $50 \%$ point on the psychometric function, which perhaps better matches the intuitive notion of "threshold of perception". Three sounds - two the same, the reference sound, and one different, the modified sound - were played in a random sequence, and the subject was asked to choose which one was different. The amount of parameter modification between the stimuli in a given trial was changed by a certain factor, the step size. The step size decreased after three correct answers and increased after one wrong answer. The test stopped after eight turnpoints. A relatively large initial step size was applied until the second turnpoint was reached, in order to allow rapid convergence toward the threshold region. After the second turnpoint the step size was reduced. The threshold was taken as the mean of the values of the amount of modification at the last six turnpoints. The values of the two step sizes, and the starting level of parameter modification, were chosen differently for each test on the basis of preliminary trials. Each trial took no more than about 10 minutes, and subjects had a break between tests.

In all tests the reference sound was kept constant, in order to increase discriminability: subjects "learnt" to recognize the reference sound. It is argued that for the purpose of relating to musical judgments, which in practice are made by musicians who may spend several hours every day concentrating on subtle details of the sound of their instrument, the threshold of most interest is the one obtained under the most favourable conditions for discrimination. It is accepted that the test circumstances are quite remote from normal musical experience, as seems to be inevitable in the design of any scientifically-acceptable test procedure, and the approach adopted seems to the authors the best that can easily be done. The 3AFC software gave visual feedback on right/wrong answers during the experiment, although some subjects performed best by keeping their eyes shut and ignoring it. Subjects did not receive any training beforehand, but if they performed erratically on the first run they were able to repeat the run. This applied only to a few subjects, and they were all able to perform the task at the second attempt. The sounds were presented diotically via Sennheiser HD580 headphones, cho-

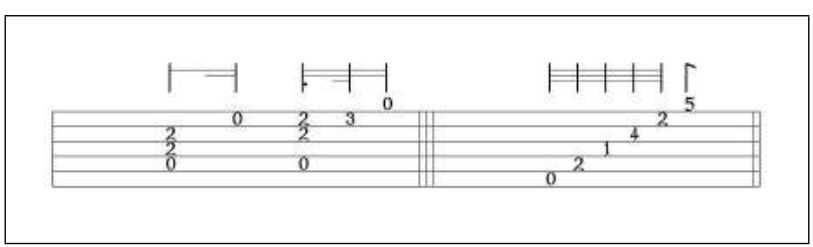

Figure 1. Guitar tablature for sound examples used in psychoacoustical tests: first the "Huntsuppe" extract, second the strummed chord with notes $\mathrm{E}_{2} \mathrm{~B}_{2} \mathrm{D}_{3} \mathrm{~B}_{3} \mathrm{CH}_{4} \mathrm{~A}_{4}$.

sen because of their diffuse field response, in a relatively quiet environment. The sampling rate was $44.1 \mathrm{kHz}$ and the number of bits was 16 .

The choice of musical input for the test sounds can have a significant influence on the JND obtained. In the first tests, on mode frequency shifting, three different sample sounds were tested: a single note (the open A string, $110 \mathrm{~Hz}$ ), a short musical fragment, and a strummed "discordant chord" with notes $\mathrm{E}_{2} \mathrm{~B}_{2} \mathrm{D \#}_{3} \mathrm{~B}_{3} \mathrm{C}_{4} \mathrm{~A}_{4}$ selected to give input at a wide range of frequencies. The musical fragment consisted of the first bar of the lute piece "The English Huntsuppe" by John Whitfelde. This and the chord are shown in tablature notation in Figure 1: the tablature applies to a guitar in regular EADGBE tuning. For the remaining tests, a single note was used for the test sound: the open A string $(110 \mathrm{~Hz})$ for the damping factor tests, and $\mathrm{C}_{3}$ on the A string $(131 \mathrm{~Hz})$ for the string parameter tests. The total stimulus length for the single notes and the chord was $1 \mathrm{~s}$, while the Huntsuppe fragment took $2 \mathrm{~s}$. These are all longer than the $300 \mathrm{~ms}$ length that yielded the lowest thresholds in the violin tests, and perhaps some advantages of echoic memory are sacrificed by using these longer stimuli. However, it was found that the sense of a realistic guitar sound was compromised if the duration was too short, and it seemed more appropriate to use the longer, more "guitarish" sounds.

The test subjects were all "musical" in one sense or another, but for the purposes of the presentation of results they are divided into two groups using the same criterion as in the work on the violin [1]: those labelled "musicians" had at least 8 years of formal training and were currently active in music-making at least once per week, while the others are labelled "non-musicians". The first author was a subject in each test, while all the other subjects were of student age. The results for the first author did not seem in any way atypical of the rest, despite his relatively advanced age.

\subsection{Physical measurements}

Physical measurements on the body and strings of several guitars are used in two different ways in this study. First, measurements are needed to calibrate the synthesis model, as has been described in detail previously [4]. Second, the same range of measurements can be analysed to give estimates of the variability in practice of the parameters for which JNDs will be found. By comparing the actual variation with the JND for perception, it is hoped to give a first 


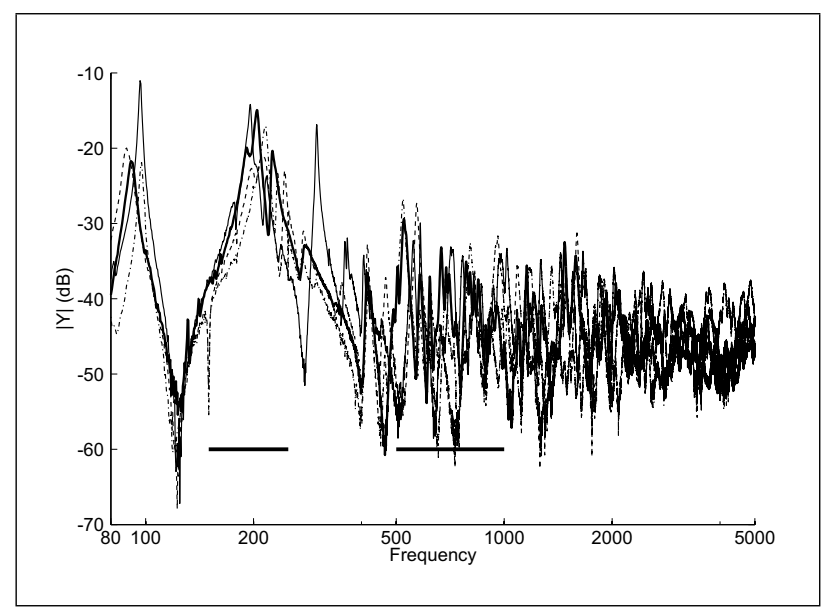

Figure 2. Magnitude of the input admittances of four guitars, measured normal to the top plate at the bridge saddle near the lowest string $\left(\mathrm{E}_{2}\right)$, plotted in decibels relative to $1 \mathrm{~m} / \mathrm{Ns}$. Heavy line: flamenco guitar by Martin Woodhouse; thin solid line: classical guitar by Michael J O'Leary; dashed line: classical guitar by Greg Smallman; dash-dot line: flamenco guitar by José Ramirez. Horizontal lines show bands used in testing: see text.

estimate of the relative sensitivity and importance of different kinds of variation in guitar construction and stringing.

Input admittance $Y(\omega)$ of the guitar body is measured by applying a controlled force either with a miniature impulse hammer (PCB 086D80) or by gently pulling a length of fine copper wire, looped around a string at the bridge saddle, until it snaps. The latter method is less accurate, but has the advantage that it can be used to apply force in different controlled directions to give information on the modal angles. In all cases the velocity response is measured with a laser-Doppler vibrometer (Polytec OFV-056), directed to a spot as close as possible to the forcing point. Signals are collected at a sampling rate of $40 \mathrm{kHz}$ by a digital data-logger based on a PC running Matlab, with a National Instruments 6220 data acquisition card. The strings of the guitar were damped during admittance measurements.

Input admittances in the direction normal to the plane of the top plate have been measured on a wide range of acoustic guitars, and a representative selection of 15 are used in the present study. Figure 2 shows results for four of these, all hand-made instruments of high quality. The heavy curve is the datum guitar used in the psychoacoustical tests, a flamenco guitar by Martin Woodhouse studied in the previous work [4]. The others are a flamenco guitar by Jose Ramirez, and classical guitars by Greg Smallman and Michael J. O'Leary. It is immediately clear that all these guitars, although different in detail, show a lot of common features. All have a strong peak in the range $80-100 \mathrm{~Hz}$, which is a Helmholtz resonance associated with the internal air cavity and the soundhole, modified by the compliance of the top and back plates. All the guitars then show a deep antiresonance (marking the frequency at which the Helmholtz resonance would occur with a rigid cavity, see equation (10.19) of Cremer [11]) followed by a cluster of strong peaks in the range $150-250 \mathrm{~Hz}$. Most then show a steady decline to a dip around $400-500 \mathrm{~Hz}$, followed by fluctuations around a rather steady level with gradually reducing peak amplitudes as the modal overlap becomes significant. One guitar (the O'Leary) has an additional strong peak around $300 \mathrm{~Hz}$ interrupting the decline to the dip. The two horizontal lines mark frequency bands that will be used in frequency-shifting tests in the next section. The first of these bands $(150-250 \mathrm{~Hz})$ covers the range where the datum guitar has its strongest peaks. The second band $(500-1000 \mathrm{~Hz})$ covers a range where the modal overlap is becoming significant, but fairly clear individual peaks can still be seen.

The other physical measurements used in this study are aimed at establishing the properties of strings. Tension and mass per unit length are readily established, but the bending stiffness and damping properties require careful measurement. Such measurements have previously been made on a typical set of nylon strings for a classical guitar, and in the course of this project corresponding measurements were made on a set of steel strings as commonly used on folk guitars. The chosen strings were Martin " $80 / 20$ Bronze". It is a familiar experience to guitarists that the sound changes a lot between when the strings are new and when they are well-played, so the strings studied here were measured in both states to give quantitative data on what changes in the ageing process.

The bending stiffness and damping properties can be estimated by analysis of sounds recorded from notes played on the guitar in the usual way [4]. The frequencies of the strongest spectral components, corresponding to "string" modes, can be best-fitted to equation (2) to yield values of $B$ and hence $E I$. The associated decay rate of each of these "string" modes can also be found using timefrequency analysis, and this gives information about the string damping. The procedure for obtaining damping information from the decay rates is a little indirect, because in most cases the decay rate is dominated by losses into the guitar body, rather than by internal damping in the string.

The approach adopted is to analyse the frequencies for a plucked note on every fret of a given string, and combine them all into a cloud plot of damping factor against frequency: examples are shown in Figure 3. This shows data for the 3rd string $\left(\mathrm{G}_{3}\right)$, for the nylon string measured previously and the new steel-cored string. The inherent damping of the string, as a function of frequency, determines the "floor level" of this cloud plot. Approximate values of $\eta_{A}, \eta_{F}, \eta_{B}$ can be estimated by fitting eqquation (4) to the trend of this floor level, as illustrated by the curves in Figure 3 . The fitting process was carried out manually, with some regard to the error tolerances of the data points. Note that the high-frequency fit line for the nylon string was influenced by additional data obtained by a different approach, not shown here: see [4] for more detail. It is clear from the figure that the nylon string has higher damping than the steel string. The measured string properties are summarised in Table I. 
Table I. Properties of guitar strings: (a) D’Addario Pro Arté "Composites, hard tension" nylon strings; (b) Martin "80/20 bronze" steel strings in new condition; (c) as (b) but in used condition.

\begin{tabular}{|c|c|c|c|c|c|c|c|c|c|}
\hline String: & & & & 1 & 2 & 3 & 4 & 5 & 6 \\
\hline Tuning note & & & & $\mathrm{E}_{4}$ & $\mathrm{~B}_{3}$ & $\mathrm{G}_{3}$ & $\mathrm{D}_{3}$ & $\mathrm{~A}_{2}$ & $\mathrm{E}_{2}$ \\
\hline Frequency & & $f_{n}$ & $(\mathrm{~Hz})$ & 329.6 & 246.9 & 196.0 & 146.8 & 110.0 & 82.4 \\
\hline Tension & $\begin{array}{l}\text { (a) } \\
\text { (b) }\end{array}$ & $T$ & $(\mathrm{~N})$ & $\begin{array}{c}70.3 \\
121\end{array}$ & $\begin{array}{c}53.4 \\
135\end{array}$ & $\begin{array}{c}58.3 \\
182\end{array}$ & $\begin{array}{c}71.2 \\
185\end{array}$ & $\begin{array}{c}73.9 \\
169\end{array}$ & $\begin{array}{c}71.6 \\
147\end{array}$ \\
\hline Mass/unit length & $\begin{array}{l}\text { (a) } \\
\text { (b) }\end{array}$ & $m$ & $(\mathrm{~g} / \mathrm{m})$ & $\begin{array}{l}0.38 \\
0.57\end{array}$ & $\begin{array}{l}0.52 \\
1.13\end{array}$ & $\begin{array}{l}0.90 \\
2.42\end{array}$ & $\begin{array}{l}1.95 \\
4.37\end{array}$ & $\begin{array}{l}3.61 \\
7.13\end{array}$ & $\begin{array}{c}6.24 \\
11.02\end{array}$ \\
\hline Bending stiffness & $\begin{array}{l}\text { (a) } \\
\text { (b) } \\
\text { (c) }\end{array}$ & $E I$ & $\left(10^{-6} \mathrm{~N} / \mathrm{m}^{2}\right)$ & $\begin{array}{l}130 \\
108 \\
103\end{array}$ & $\begin{array}{l}160 \\
336 \\
394\end{array}$ & $\begin{array}{l}310 \\
234 \\
343\end{array}$ & $\begin{array}{c}51 \\
255 \\
418\end{array}$ & $\begin{array}{c}40 \\
327 \\
480\end{array}$ & $\begin{array}{c}57 \\
667 \\
719\end{array}$ \\
\hline Inharmonicity & $\begin{array}{l}\text { (a) } \\
\text { (b) } \\
\text { (c) }\end{array}$ & $B$ & $\left(10^{-6}\right)$ & $\begin{array}{l}43 \\
21 \\
20\end{array}$ & $\begin{array}{l}70 \\
58 \\
68\end{array}$ & $\begin{array}{c}124 \\
30 \\
44\end{array}$ & $\begin{array}{l}17 \\
32 \\
53\end{array}$ & $\begin{array}{l}13 \\
45 \\
66\end{array}$ & $\begin{array}{c}19 \\
106 \\
114\end{array}$ \\
\hline \multirow[t]{3}{*}{ Loss coefficients } & $\begin{array}{l}\text { (a) } \\
\text { (b) } \\
\text { (c) }\end{array}$ & $\eta_{F}$ & $\left(10^{-5}\right)$ & $\begin{array}{c}40 \\
7 \\
6\end{array}$ & $\begin{array}{c}40 \\
6 \\
7\end{array}$ & $\begin{array}{l}14 \\
11 \\
17\end{array}$ & $\begin{array}{c}5 \\
9 \\
33\end{array}$ & $\begin{array}{c}7 \\
11 \\
17\end{array}$ & $\begin{array}{c}2 \\
5 \\
10\end{array}$ \\
\hline & $\begin{array}{l}\text { (a) } \\
\text { (b) } \\
\text { (c) }\end{array}$ & $\eta_{B}$ & $\left(10^{-2}\right)$ & $\begin{array}{l}2.4 \\
0.7 \\
0.7\end{array}$ & $\begin{array}{l}2.0 \\
1.1 \\
1.1\end{array}$ & $\begin{array}{l}2.0 \\
1.8 \\
6.8\end{array}$ & $\begin{array}{l}2.0 \\
1.1 \\
4.1\end{array}$ & $\begin{array}{l}2.5 \\
0.4 \\
2.9\end{array}$ & $\begin{array}{l}2.0 \\
0.3 \\
3.8\end{array}$ \\
\hline & $\begin{array}{l}\text { (a) } \\
\text { (b) } \\
\text { (c) }\end{array}$ & $\eta_{A}$ & $(1 / s)$ & $\begin{array}{l}1.5 \\
2.5 \\
1.5\end{array}$ & $\begin{array}{l}1.2 \\
1.0 \\
1.0\end{array}$ & $\begin{array}{l}1.7 \\
1.2 \\
0.6\end{array}$ & $\begin{array}{l}1.2 \\
1.3 \\
0.7\end{array}$ & $\begin{array}{l}0.9 \\
1.2 \\
0.6\end{array}$ & $\begin{array}{l}1.2 \\
1.7 \\
1.4\end{array}$ \\
\hline
\end{tabular}

The differences between the nylon and steel strings in new condition are broadly as one would expect. The nylon strings have lower tensions and a different pattern of bending stiffness because of the difference in construction: for the nylon set the top three strings are monofilaments while the lower three have multifilament cores with metal windings. The steel strings have monofilament cores throughout, and only the top two strings are not wrapped. The monofilament nylon strings have higher internal damping than the steel, but the difference is much less marked for the lower three strings.

The effect of age and use on the steel strings can also be seen. Previous investigators have reported increased damping between new and old guitar strings [12, 13], but without sufficient detail to allow a fit to equation (4). It is generally accepted that the mechanism for this ageing effect is the accumulation of dirt and corrosion products between the windings of wrapped strings [13], perhaps with some additional cumulative wear of the outer wrappings where the strings make contact with the frets. The measurements reported here support this picture: there is very little change in the two highest strings, which are not wrapped. For the wrapped strings there is a marked increase in damping, especially in $\eta_{B}$ which determines the decay at the highest frequencies.

Also, not noted by previous authors, there is a marked increase in the effective bending stiffness of some of the strings. This seems perfectly credible since the contact conditions between the windings in the tensioned string will contribute to the bending stiffness, and material col- lecting between the windings will surely change those conditions. This is consistent with the observed increase in $\eta_{B}$, which is the loss factor associated with the bending stiffness term in the governing equation. It may be noted that the bending stiffness values given here are in good general agreement with values reported by Järveläinen $e t$ al. [7], noting that their steel-string results match the "used" results more closely than the "new" results. Their paper does not report the condition of the strings as measured.

\section{Results for properties of the guitar body}

\subsection{Psychoacoustical tests}

The first series of psychoacoustical tests concerned the shifting of all body mode frequencies by a given factor, keeping other simulation parameters fixed. This modification is of course somewhat artificial, but a possible physical interpretation will be discussed in the next subsection. The datum for this test was based on the modal properties of the flamenco guitar by Martin Woodhouse. This test was run three times, using the three different musical inputs described above. The three tests were completed by 8 musicians and 5 non-musicians (according to the criterion described above). The results are summarised in Figure 4. The program that runs the $3 \mathrm{AFC}$ protocol gives as output the mean and standard deviation over the range defined by the final 6 turnpoints. The plots show, for each test subject, the mean on the horizontal axis and the relative standard deviation (standard deviation divided by mean) on the vertical axis. The numbers on the horizontal axis indicate the 


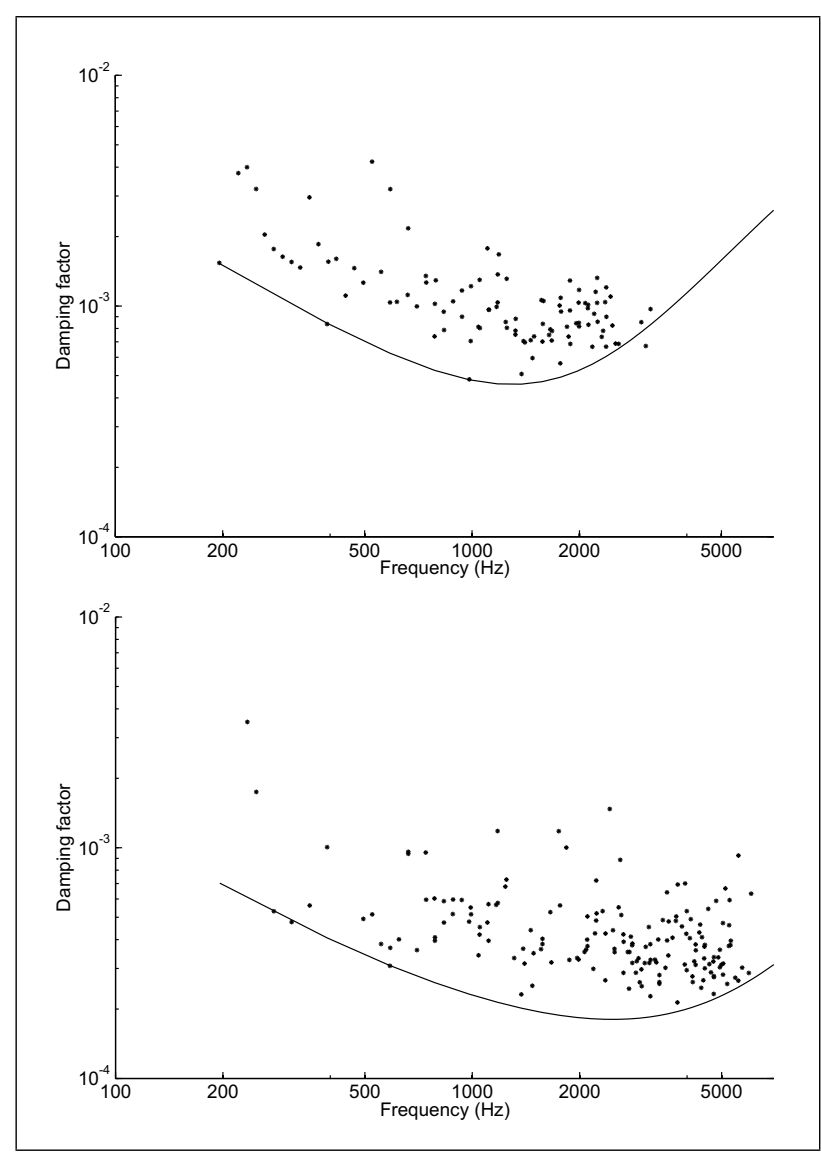

Figure 3. Damping measurements on (top) a nylon guitar string (3rd string, $\mathrm{G}_{3}$ ) and (bottom) the corresponding string from a steel-cored set. Discrete points show individual measured decay rates, curves show fits to the floor level using equation (4).

percentage shift in mode frequency. Musicians are indicated by stars, non-musicians by plus symbols. The averages over all test subjects are shown by the open circles.

The open square symbols show the average of the four subjects who achieved the lowest mean levels. As was suggested earlier, within the scope of the present study the most interesting threshold to compare with serious musical judgments is that achieved by the best listeners under the most favourable conditions. In the violin tests, the best five results were presented for this purpose [1]. In the tests reported here the number of subjects is sometimes rather low, and it was decided to average the best four. The numerical results of this best-four measure for all the 3AFC tests presented here are given in the figure captions.

It can be seen that for each of the three musical inputs the best subjects could discriminate a shift of $1 \%$ or less. Interesting patterns can be seen in the detailed results, despite the small number of subjects that would make any elaborate statistical analysis of limited value. The general spread of results, and the two averages, look very similar for the single note and the Huntsuppe extract. For the single note the musicians and non-musicians are intermingled, while for Huntsuppe all but one of the non-musicians has a worse result than all the musicians. Perhaps nonmusicians have their performance degraded by informa-

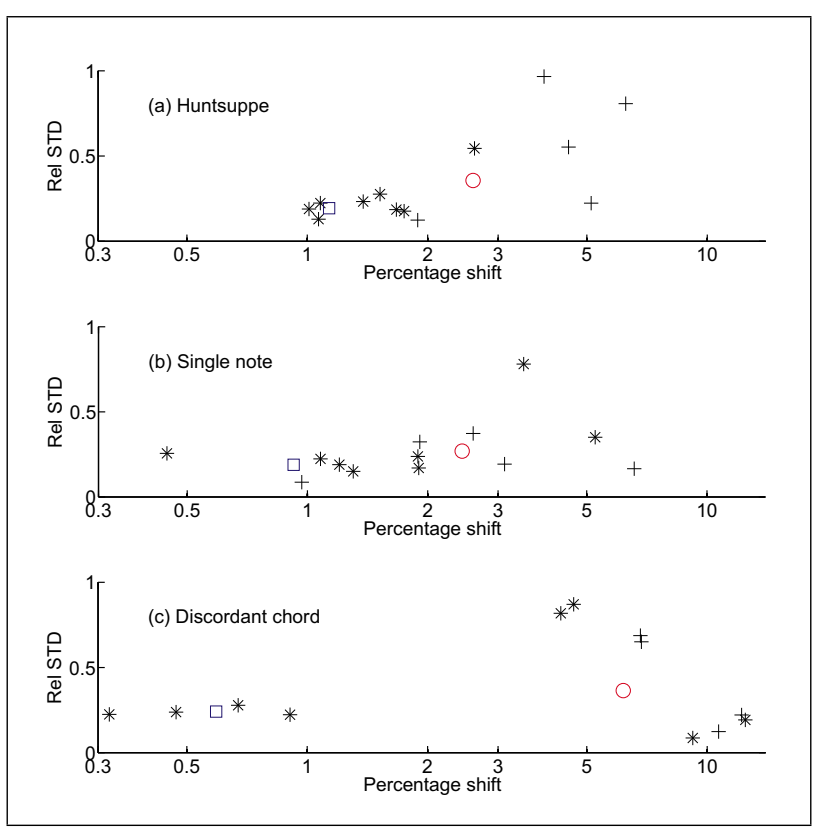

Figure 4. Results from 3AFC studies for shifting all mode frequencies of the datum guitar, using as input (a) the Huntsuppe fragment; (b) a single note (open A string); (c) the discordant chord. Horizontal axis shows percentage frequency shift on a logarithmic scale, vertical axis shows relative standard deviation. Points $*$ denote musicians, points + denote non-musicians. Open circle: average of all subjects; open square: average of best four subjects. The percentage shifts corresponding to the best-four averages are (a) 1.13 ; (b) 0.92 ; (c) 0.59 .

tional masking while experienced musicians do not, with such an obviously musical sound sample as "Huntsuppe".

The chord reveals a different picture. Four of the musicians achieved very low thresholds with this sound input, but all other subjects seem to have been seriously distracted by this rather non-musical sound, and achieved only rather high thresholds. One of the musicians comes out worst of all in this test, whereas for Huntsuppe all the musicians performed well. This is a striking example of informational masking, especially since the group of four subjects who did well actually produced a lower average than in either of the other tests. That is as one would hope: this signal contains, deliberately, a wider range of frequency components so that analytical listeners with sufficient skill and experience to take advantage of it can make very subtle judgements down to $0.32 \%$ shift in the best case. This corresponds to shifting the mode frequencies about 6 cents or $1 / 20$ of a semitone, an impressively small amount that is not very much bigger than the threshold for pitch discrimination tasks (see e.g. [14]).

The thresholds are of the same order as the "best five" thresholds in the corresponding violin tests, but the detailed pattern seems different. The choice of input had a more marked effect in the violin, and to achieve the lowest thresholds a very short, and consequently rather unmusical, sound sample was used.

The best-performing 7 subjects of the first test series were asked to undertake two further tests, in which fre- 
quencies were shifted only in limited bands. The two bands studied were shown in Figure 2, and this time the only input sound tested was the single-note open A string. The results of the tests are shown in Figure 5 in the same format as Figure 4. Rather unexpectedly, the "best four" threshold for the first band, containing the strongest peaks of the datum guitar, was much higher than for the second band. Related tests for the violin, using the so-called "Dünnwald bands", gave thresholds around 2\% for the first three bands (see Figure 6 of Fritz et al. [1]). Band 1 in the present study gave a comparable result, but band 2 gave a lower result than was observed in any of the violin tests. The best-four JND in this case is virtually the same as for the corresponding test when all frequencies were shifted. Further studies might be worth conducting to add more detail to this observation.

The final pair of tests relating to the perception of body mode changes investigated the effect of damping factors. This time the mode frequencies were kept fixed but the modal $\mathrm{Q}$ factors were all scaled by a given factor: $\mathrm{Q}$ factor is the inverse of damping factor, so high $\mathrm{Q}$ corresponds to low damping. In two separate tests the $\mathrm{Q}$ factors were increased and decreased, to look for any asymmetry in the perception. Thirteen test subjects took these two tests, 10 musicians and 3 non-musicians. Results are shown in Figure 6 in the same format as Figs. 4 and 5, except that the numbers on the horizontal axis now indicate the scale factor applied rather than the percentage change. This means that "no change" corresponds to the value 1 rather than 0 as in the previous results, which has the effect of making the relative standard deviations numerically smaller.

An asymmetry is seen between the two tests, but it does not significantly affect the threshold value for the best four listeners. They achieved a value around 1.2, i.e. a $20 \%$ change in Q factors, in both cases. However, across the whole set of test subjects the spread is much greater when the $\mathrm{Q}$ factors were decreased compared to the increasing case. Many participants reported initial difficulty in hearing the difference when Q factors were decreased, although once they had learned to recognise the sound change they often achieved quite low thresholds.

It was not thought worthwhile to test separate frequency bands in this case, for two reasons. First, body damping is most readily altered by a physical change of material, and this is likely to have an effect over a broad frequency range. Second, it will be shown shortly that the JND for perception of a change in body damping is of the same order as the measured standard deviation among real guitars, so that the perceptual significance is probably marginal and digging deeper into the details is likely to be of limited importance.

\subsection{Physical measurements}

The next step is to look at physical measurements to assess how these thresholds of perception compare with the actual extent of variation between guitars. Of course, one would never find two real instruments which differed by having all the body frequencies simultaneously scaled by

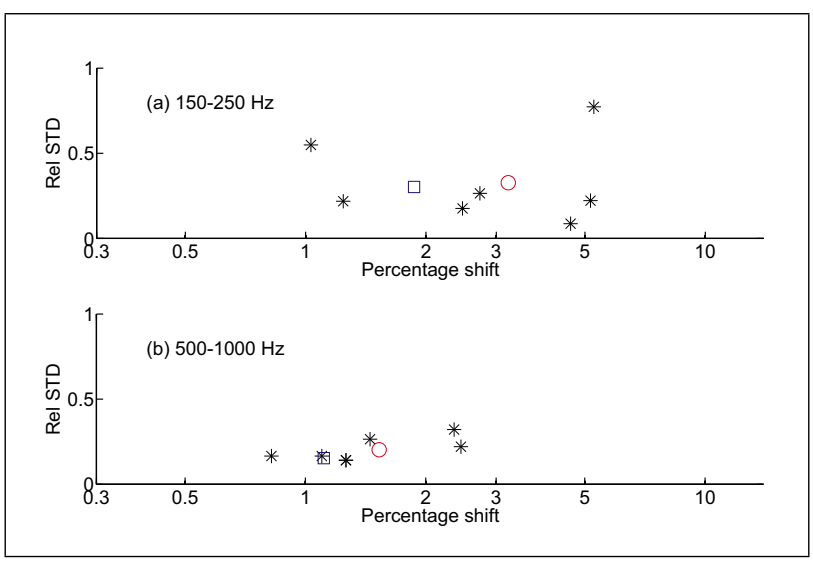

Figure 5. Results in the format of Figure 4 from 3AFC studies for shifting all mode frequencies of the datum guitar lying in (a) the band $150-250 \mathrm{~Hz}$; and (b) the band $500-1000 \mathrm{~Hz}$, using as input a single note (open A string). The percentage shifts corresponding to the best-four averages are (a) 1.87 ; (b) 1.53 .

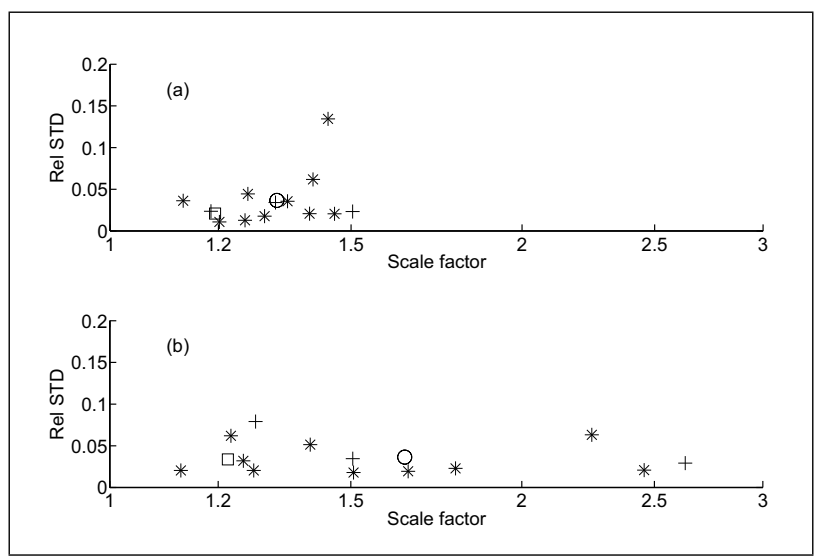

Figure 6. Results in the format of Figure 4 from 3AFC studies for scaling all modal Q factors of the datum guitar (a) upwards; and (b) downwards, using as input a single note (open A string). Horizontal axis shows scale factor applied by multiplication or division to $\mathrm{Q}$ factors. The scale factors corresponding to the bestfour averages are (a) 1.19; (b) 1.22.

exactly the same factor. However, it is easy to visualise a change which would in principle have this effect: it would happen if the dimensions of the guitar were all scaled, keeping the same materials and other details. Some simple deductions can then be drawn by scaling analysis. If the main vibrating elements of the guitar body are treated as thin, flat plates deforming in bending, then the natural frequencies scale proportional to $h / d^{2}$ where $d$ is the scale factor for dimensions in the plane and $h$ is the scale factor for thickness. Taking $1 \%$ as a round number for the order of magnitude of the threshold of detection for shifting frequencies, it follows that the JNDs for dimensional scaling are approximately $1 \%$ if $d$ and $h$ are both scaled together (full geometric scaling), 1\% again if thickness is changed keeping the in-plane dimensions fixed, and only $0.5 \%$ if in-plane dimensions are scaled keeping the thickness the same. These are all very small numbers: they amount to changing in-plane dimensions no more than a few $\mathrm{mm}$, and 


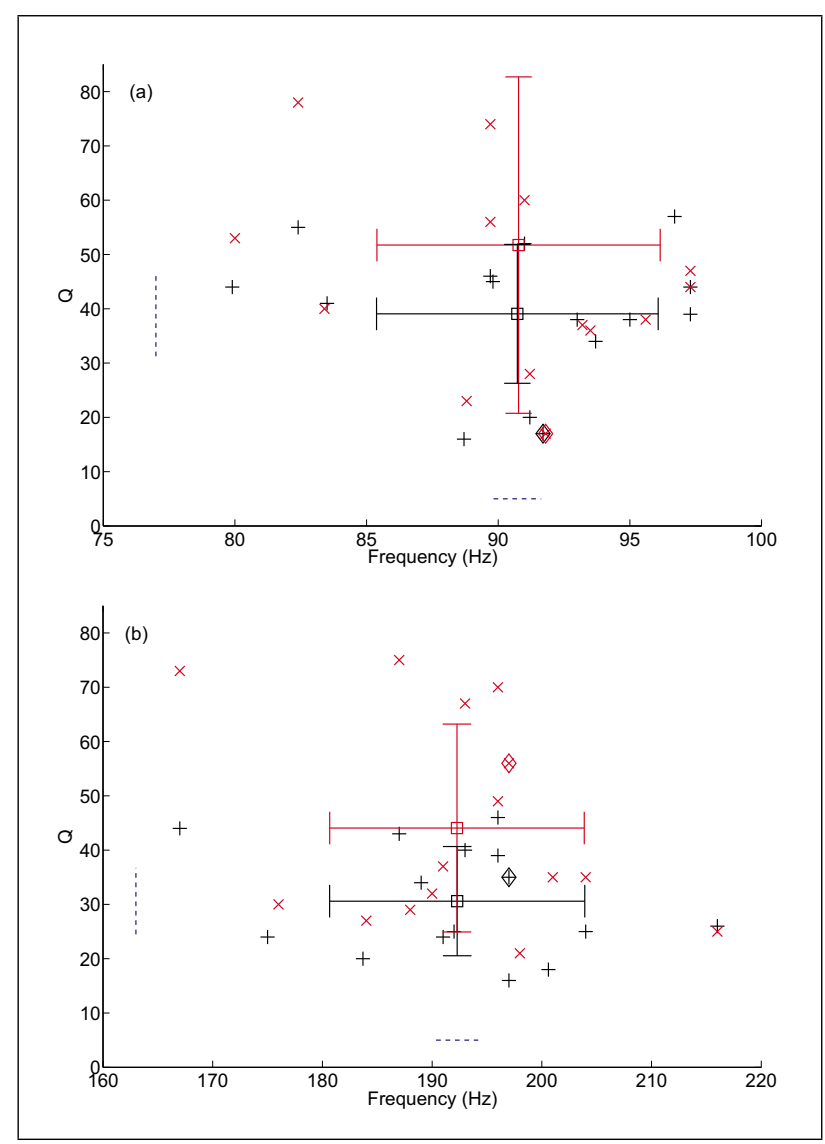

Figure 7. Modal frequencies and Q factors for 15 guitars, for the highest peak in the frequency bands (a) $75-100 \mathrm{~Hz}$, the "Helmholtz resonance"; (b) $150-250 \mathrm{~Hz}$, the "first top plate mode". Plus and cross symbols show fits without and with compensation for string damping, respectively. Diamond symbols mark the datum guitar for the listening tests. Open squares show the means, and range bars show one standard deviation above and below the means, for the two sets of fitted data. The ranges for the compensated results can be recognised by their higher Q values. Dashed lines near the axes show approximately $1 \mathrm{JND}$ above and below the uncompensated means from the results of section 4.1 .

to changing a typical top plate thickness by a mere $25 \mu \mathrm{m}$. Real instruments have dimensional variations which are considerably bigger than this, especially in thickness, so on this ground alone it is no surprise that guitars can sound different.

The simplest approach to obtaining quantitative information from admittance measurements on a set of guitars is to perform explicit modal analysis. This can only be expected to give reliable results at relatively low frequencies: pole-residue extraction, whatever the method, becomes sensitive once the modal overlap begins to approach unity. To illustrate the kind of values which emerge from such an analysis, Figure 7 shows results for modal frequency and Q factor for 15 guitars, for the highest peak in each of the two low-frequency bands mentioned in the discussion of Figure 2: 75-100 Hz, the "Helmholtz resonance", and $150-250 \mathrm{~Hz}$, the "first top plate mode".
A complicating factor needs to be noted. The admittance measurements are made with the strings well damped, because the purpose is to obtain information about the body behaviour without the effect of the strings. However, the damped strings remove some energy from the body vibration, and this will tend to reduce the measured Q factors. This effect can be compensated, at least approximately. The damped strings will act essentially as semi-infinite strings and will thus present an impedance to the bridge which is real and, if the strings are all assumed to be attached at the same point, equal to the sum of the string impedances given by $Z=\sqrt{T m}$. From the data for nylon strings in Table I, the sum of these impedances is $2.12 \mathrm{Ns} / \mathrm{m}$. To compensate, the inverse of $Y(\omega)$ is taken, this real quantity is subtracted, then the result re-inverted to give a new admittance. This approach can be expected to work well at higher frequencies, but it is hard to be sure if the strings are well enough damped at low frequencies to justify the semi-infinite approximation.

Figure 7 shows results before and after this compensation. For each case the mean and scatter are indicated by lines showing one standard deviation above and below the mean for frequency and Q. Interestingly, the standard deviations have a similar pattern for both cases. The relative standard deviations for frequency are $5.5 \%$ and $6 \%$ for the two cases, and those for Q factor about $33 \%$ in both cases before compensation.

To obtain good comparative information about modal frequencies over a broader bandwidth is more challenging. It is not possible to identify the "same" mode in different guitars except at very low frequencies: sensitivity to small structural changes increases with frequency, as is familiar in other vibration problems (see e.g. [15]). For mode frequencies, the threshold for audible changes is so small that it is hard to obtain any measure which is good enough: scaling frequencies by $1 \%$ means that one guitar body might have 101 modes in a frequency range where another guitar had 100, and there is no known analysis method which can give robust discrimination at this level. Any measure which can be computed shows much bigger variability than this, and there is always a strong suspicion that it is revealing the vagaries of the analysis method more than the real differences between guitars.

Things are a little more promising in the case of damping estimation over a wide frequency range. Taking the inverse FFT of an input admittance gives an impulse response function of the guitar body, and this can be processed using time-frequency analysis to give a sonogram representation. In each frequency band, a best-fitting exponential decay can then be obtained. Combining results from several different choices of the time/frequency resolution tradeoff improves the robustness of the results. The process is described in more detail elsewhere [16]. Some results are shown in Figure 8: compensation for string damping has been included. In Figure $8 \mathrm{a}$ the discrete points represent, for the datum guitar, frequency/decay combinations which satisfied an assumed criterion for good fit quality. For compatibility with other plots, decay 


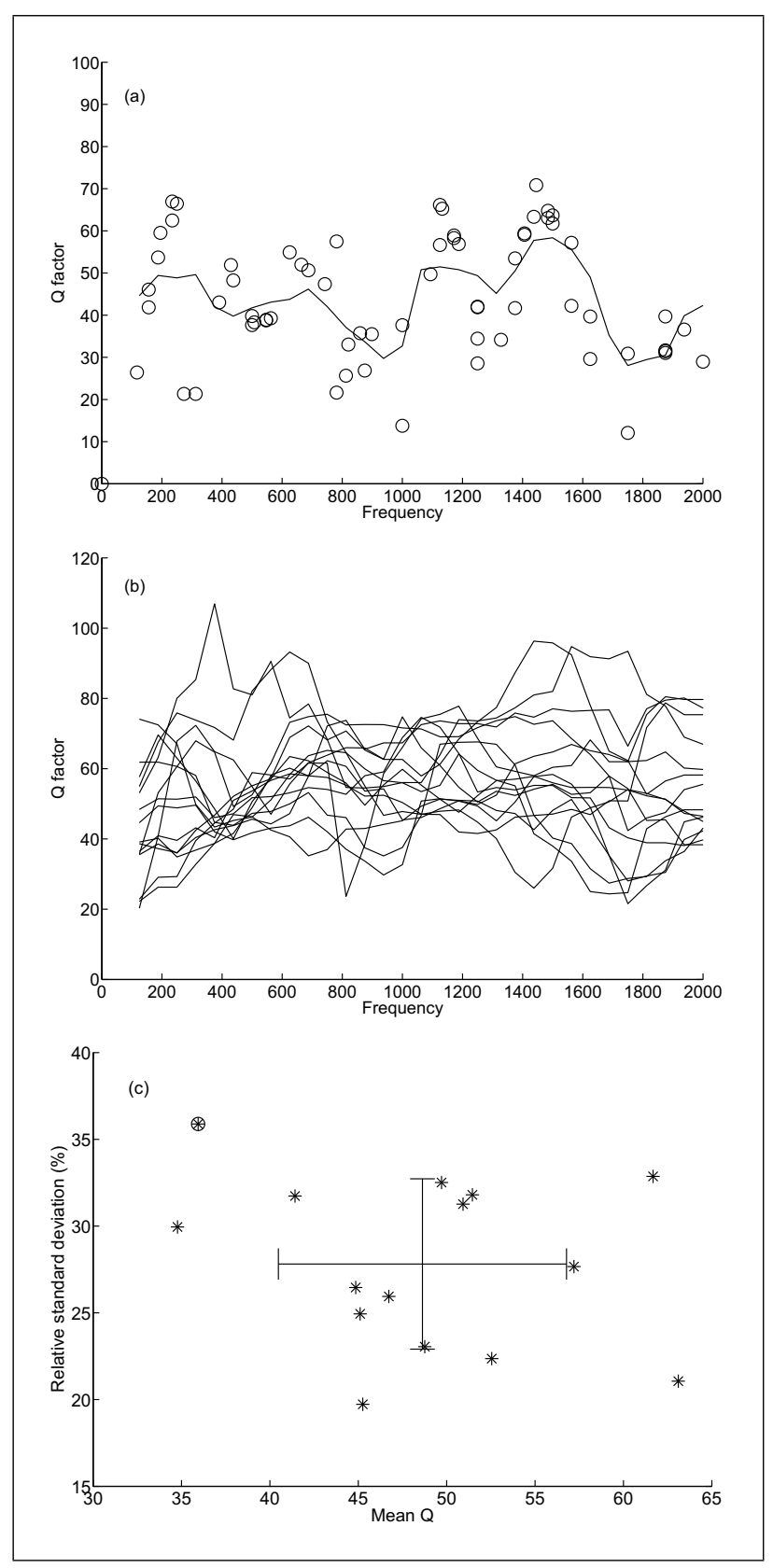

Figure 8. Damping trends for guitar bodies determined from time-frequency analysis. (a): individual measurements of frequency and equivalent $\mathrm{Q}$ factor (circles) and the band average of these (solid line) for the datum guitar; (b): the solid lines as in (a), for the set of 15 guitars; (c) the mean and standard deviation of the results from (b) across the frequency range, with range bars indicating one standard deviation above and below the mean on each axis. The circle marks the datum guitar for the listening tests.

rate is represented as an equivalent $\mathrm{Q}$ factor. The continuous line is simply a moving band average of these discrete points.

Figure $8 \mathrm{~b}$ then shows the band-averaged lines for the 15 guitars in the test set. Finally, Figure 8c shows a scatter plot of the mean and standard deviation of these bandaveraged lines for the 15 guitars. As in Figure 7, the large cross represents one standard deviation above and

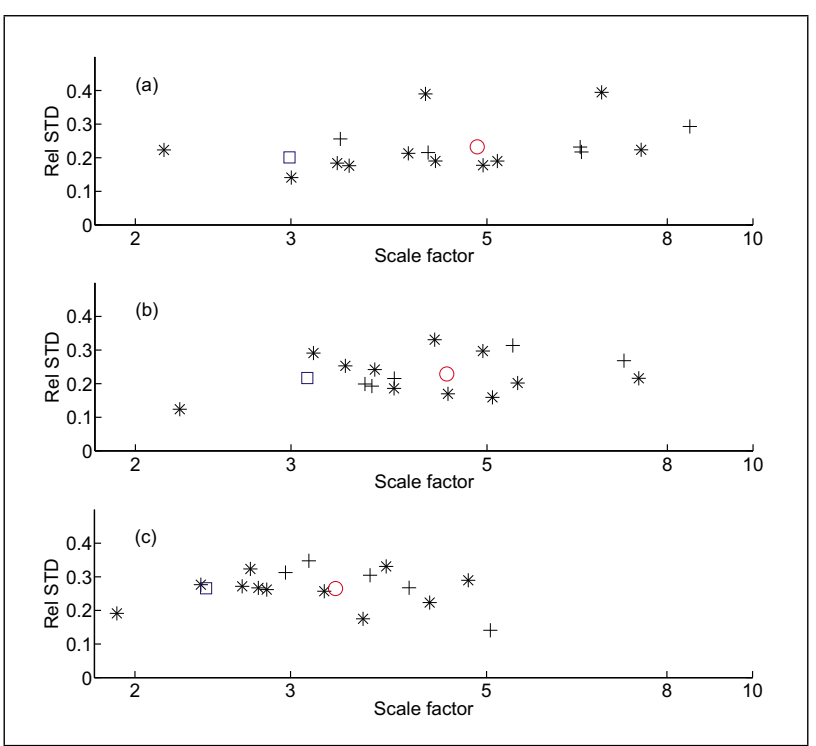

Figure 9. Results in the format of Figure 4 from 3 AFC studies for changing the damping parameters of the 5th string, based on datum values for the new steel strings. Case (a): varying $\eta_{B}$, test note $\mathrm{E}_{2}$; (b): varying $\eta_{B}$, test note $\mathrm{C}_{3}$; (c): varying $\eta_{F}$, test note $\mathrm{C}_{3}$. The scale factors corresponding to the best-four averages are (a) 2.99; (b) 3.13 ; (c) 2.41 .

below the mean on each axis. The main result is that this frequency-averaged measure of damping has a relative standard deviation of the order of $20 \%$, comparable to the JND found earlier for perception of damping changes. This suggests that the extremes of mean damping in the measured set of guitars are sufficiently different that the effect should be audible, but the results do not lead one to expect that this will be a very strong component of the perception of tonal differences among guitars.

\section{Results for properties of the strings}

The final set of JND experiments relates to changes in string properties, including those properties that changed as the string aged: one objective was to find out which of those changes are likely to contribute most to the perception of ageing. Using as datum the properties of the new steel strings listed in Table I, changes to the damping properties of the 5th string were investigated using singlenote test sounds. These 3AFC tests were completed by 11 musicians and 5 non-musicians. One test was also conducted relating to changes in the bending stiffness of this string: it gave results in line with those of Järveläinen $e t$ al. [7] and the details are not reproduced here. The actual bending stiffness of this string was found to be close to the threshold for detecting an increase in bending stiffness when starting from a much lower level. In the aged string, the bending stiffness only increased by a factor 1.6 (see Table I). If that change is audible at all the effect is likely to be small.

The results for the damping tests are shown in Figure 9, in a similar format to earlier $3 \mathrm{AFC}$ results. As noted earlier, the perceptual effects of changing $\eta_{A}$ for this string 
were found to be so weak that it was not possible to conduct a meaningful test. However, both $\eta_{B}$ and $\eta_{F}$ gave clear results. The parameter $\eta_{B}$, associated with bending stiffness and mainly affecting higher frequencies, was tested using two different notes "played" on the same string. The results are shown in Figures 9a,b, and show a good level of consistency between the two notes. The spread, overall mean and best-four mean are all very similar for both cases. The best listeners need about a factor 3 increase in this parameter to perceive the change. Note from Table I that in the aged string this parameter increased from 0.004 to 0.029 : that change should be very clearly audible.

Figure 9c shows corresponding results for $\eta_{F}$. All subjects gave a somewhat lower threshold for this parameter, compared to $\eta_{B}$, and the very best listener achieved a JND of less than a factor 2 . In the aged string $\eta_{F}$ increased in the ratio 17:11 for this string, from Table I. This effect is probably not audible to most listeners. It can be concluded that the factor most likely to account for the difference in sound of the aged string is $\eta_{B}$.

\section{Conclusions}

Psychoacoustical experiments have been reported to establish JND levels for the perception of changes to various parameters of a model of synthesised guitar sound [3, 4]. These investigations parallel earlier work exploring related JNDs for violin sounds [1]. The parameters investigated here relate to the behaviour of the guitar body (modal frequencies and damping factors) and of the strings (bending stiffness and damping model parameters). All tests were carried out using a three-alternative forced-choice methodology. It is argued that for application to musical judgements, which can be very subtle, the most interesting perceptual thresholds are those attainable by the best listeners under the most favourable conditions. To give an estimate of these, the results for the best four subjects in each test were averaged.

Results of physical measurements have also been reported, to give an indication of the spread of each parameter among real guitar bodies or strings. A comparison with the psychoacoustical results gives a first impression of which quantities might be responsible for the biggest perceptual differences between instruments. By far the most sensitive parameter of all those tested relates to shifting the frequencies of body modes. The best four listeners could perceive a $1 \%$ shift when all frequencies were scaled together, a small change compared to the actual variation among guitars. This performance was only degraded slightly when the shifted frequencies were band-limited in two different bands tested. The results were of the same order as the corresponding JNDs found for violin sounds [1]: if anything, the JNDs seem to be somewhat lower for the guitar sounds, perhaps because the fully-coupled synthesis method gives the listener additional auditory cues associated with transient decay rates of the string overtones. Body mode damping has to be changed by about $20 \%$ for perception according to the definition used here. Actual variations of modal damping exceed this level, but not by a large factor, so body damping is probably quite a minor contributor to tonal perception.

String damping parameters are even less sensitive: they have to be changed by a factor of the order of 3 . However, this large factor does not mean that the effects are never heard. Measurements have been presented on strings of different materials, and on steel-cored strings in new and "aged" conditions. The damping parameters vary between these cases by factors significantly bigger than the JNDs. The results strongly indicate that the most important parameter for the perception of the degradation of sound in well-used strings is $\eta_{B}$, the damping factor associated with the bending stiffness of the string. This parameter increases greatly with age of strings, probably from the damping effect of dirt and corrosion products accumulating between the windings of the lower strings of the guitar [13]. Strings without over-winding do not deteriorate in sound to anywhere near the same extent.

The correct interpretation of the high sensitivity to shifting body frequencies is not obvious. The actual change investigated here, moving all frequencies in step, is very artificial. Listeners might be responding to the actual frequencies, or perhaps to the change in modal density (the inverse of the mean spacing between resonant frequencies), or perhaps to the shifting amplitude envelope of the body response. Modal amplitudes were not changed when the frequencies were scaled, so the amplitude pattern moves along the frequency axis with the scaling. It would require further testing to establish whether one of these factors dominates or whether all contribute significantly. Such testing would be a good target for future research, to bring the results closer to the detailed concerns of instrument makers.

\section{Acknowledgement}

The authors thank Brian Moore, Ian Cross and Alan Blackwell for many helpful discussions on this work, and Martin Woodhouse, Stewart French, Ian Cross and James Westbrook for providing access to guitars for testing. Two anonymous reviewers are thanked for constructive suggestions.

\section{References}

[1] C. Fritz, I. Cross, B. C. J. Moore, J. Woodhouse: Perceptual thresholds for detecting modifications applied to the acoustical properties of a violin. J. Acoust. Soc. Am. 122 (2007) 3640-3650.

[2] J. Woodhouse, P. Galluzzo: The bowed string as we know it today. Acta Acustica united with Acustica 90 (2004) 579589.

[3] J. Woodhouse: On the synthesis of guitar plucks. Acta Acustica united with Acustica 90 (2004) 928-944.

[4] J. Woodhouse: Plucked guitar transients: comparison of measurements and synthesis. Acta Acustica united with Acustica 90 (2004) 945-965.

[5] H. A. K. Wright: The acoustics and psychoacoustics of the guitar. PhD Thesis, Cardiff University, 1996.

[6] H. Järveläinen, V. Välimäki, M. Karjalainen: Audibility of the timbral effects of inharmonicity in stringed instrument tones. Acoustics Research Letters Online 2 (2001) 79-84. 
[7] H. Järveläinen, M. Karjalainen: Perceptibility of inharmonicity in the acoustic guitar. Acta Acustica united with Acustica 92 (2006) 842-847.

[8] D. Ewins: Modal testing: Theory, practice and application. Research Studies Press, 2000.

[9] C. Valette: The mechanics of vibrating strings. - In: Mechanics of Musical Instruments. A. Hirshberg, J. Kergomard, G. Weinreich (eds.). Springer-Verlag, Vienna, 1995.

[10] H. Levitt: Transformed up-down methods in psychoacoustics. J. Acoust. Soc. Am. 49 (1971) 467-477.

[11] L. Cremer: The physics of the violin. MIT Press, Cambridge MA, 1984.
[12] J. B. Allen: On the aging of steel guitar strings. Catgut Acoust. Soc. Newsletter 26 (1976) 27-28.

[13] R. J. Hanson: Analysis of "live" and "dead" guitar strings. J. Catgut Acoust. Soc. 48 (1987) 10-16.

[14] B. C. J. Moore: An introduction to the psychology of hearing. Elsevier Academic Press, London, 2004.

[15] C. S. Manohar, A. J. Keane: Statistics of energy flows in spring-coupled one-dimensional subsystems. Phil. Trans. Royal Soc. London A 346 (1994) 525-542.

[16] J. Woodhouse, R. S. Langley: Interpreting the input admittance of violins and guitars. Acta Acustica united with Acustica (2012) in press. 